\title{
Study of nonlinear vibration of Euler-Bernoulli beams by using analytical approximate techniques
}

\section{Abstract}

In this paper, nonlinear responses of a clamped-clamped buckled beam are investigated. Two efficient and easy mathematical techniques called He's Variational Approach and Laplace Iteration Method are used to solve the governing differential equation of motion. To assess the accuracy of solutions, we compare the results with the Runge-Kutta 4th order. The results show that both methods can be easily extended to other nonlinear oscillations and it can be predicted that both methods can be found widely applicable in engineering and physics.

\section{Keywords}

Nonlinear Vibration; Laplace Iteration Method; He's Variational Approach; Euler-Bernoulli beam.

\author{
S. Bagheri, A. Nikkar ${ }^{*}$, \\ H. Ghaffarzadeh \\ Faculty of Civil Engineering, University of \\ Tabriz, Tabriz, Iran. \\ Received 02 Mar 2013 \\ In revised form 09 Apr 2013 \\ *Author email: ali.nikkar@yahoo.com
}

\section{INTRODUCTION}

The Euler-Bernoulli theory of beams provides a reasonable explanation of the bending behavior of long isotropic beams. It is based on the assumption that a relationship between bending moment and the beam curvature exists. The vibration problems of uniform Euler-Bernoulli beams can be solved by analytical or approximate approaches. In this respect, several methods have been developed recently for finding approximate solutions to nonlinear problems; see (Boukhalfa and Hadjoui, 2010; Da Silva et al., 2009; He, 2006; Ganji et al., 2011; Ganji, 2012; Bayat et al., 2011; Khan et al., 2012; Nikkar et al., 2012; Ghasempoor et al., 2102; Ghotbi et al., 2012; Fereidoon et al., 2010) and the references therein.

Lai et al. (2008) utilized Adomian Decomposition Method to obtain the natural frequencies and mode shapes for the Euler- Bernoulli beam under various supporting conditions. The single-mode discretization was employed to solve free vibrations of a simply supported buckled beam by Burgreen (1984). Pirbodaghi et al. (2009) studied non-linear vibration behavior of geometrically non-linear Euler-Bernoulli beams subjected to axial loads using homotopy analysis method. Also, 
the effect of vibration amplitude on the non-linear frequency and buckling load is discussed. Hsu et al. (2008) used Modified Adomian Decomposition Method to solve free vibration of non-uniform Euler-Bernoulli beams with general elastically end conditions. Liu and Gurram (2009) investigated the free vibration of Euler-Bernoulli beam under various supporting conditions using a variational iteration method (VIM). At the end, recently, Barari et al. (2011) studied non-linear vibration behavior of geometrically non-linear Euler-Bernoulli beams using variational iteration method and parameter perturbation method.

The Laplace Iteration Method (LIM) and Variational Approach (VA) which were introduced by Rafieipour et al. (2012) and He (2007) are very powerful methods in solving non-linear differential equations and studying nonlinear vibration of beams. The Variational Approach which is also called He's Variational Approach, as well as the Laplace Iteration Method, was utilized here to obtain the analytical expression for geometrically non-linear vibration of clamped-clamped Euler-Bernoulli beams fixed at one end. Geometric non-linearity arises from non-linear strain-displacement relationships. This type of nonlinearity is most commonly treated in the literature. Sources of this type of nonlinearity include midplane stretching, large curvatures of structural elements, and large rotation of elements.

\section{MATHEMATICAL FORMULATION}

Consider a straight beam on an elastic foundation with length $\boldsymbol{L}$, a cross-section $\boldsymbol{A}$, a mass per unit length $\boldsymbol{\mu}$, moment of inertia $\boldsymbol{I}$, and modulus of elasticity $\boldsymbol{E}$ that subjected to an axial force of magnitude $\boldsymbol{F}^{\prime}$ as shown in Fig. 1. It is assumed that the cross-sectional area of the beam is uniform and its material is homogenous. The beam is also modeled according to the Euler Bernoulli beam theory. Planes of the cross sections remain planes after deformation, straight lines normal to the midplane of the beam remain normal, and straight lines in the transverse direction of the cross section do not change length. The first assumption ignores the in plane deformation. The second assumption ignores the transverse shear strains and consequently the rotation of the cross section is due to bending only. The last assumption, which is called the incompressibility condition, assumes no transverse normal strains. The last two assumptions are the basis of the Euler-Bernoulli beam theory (Rao, 2007).

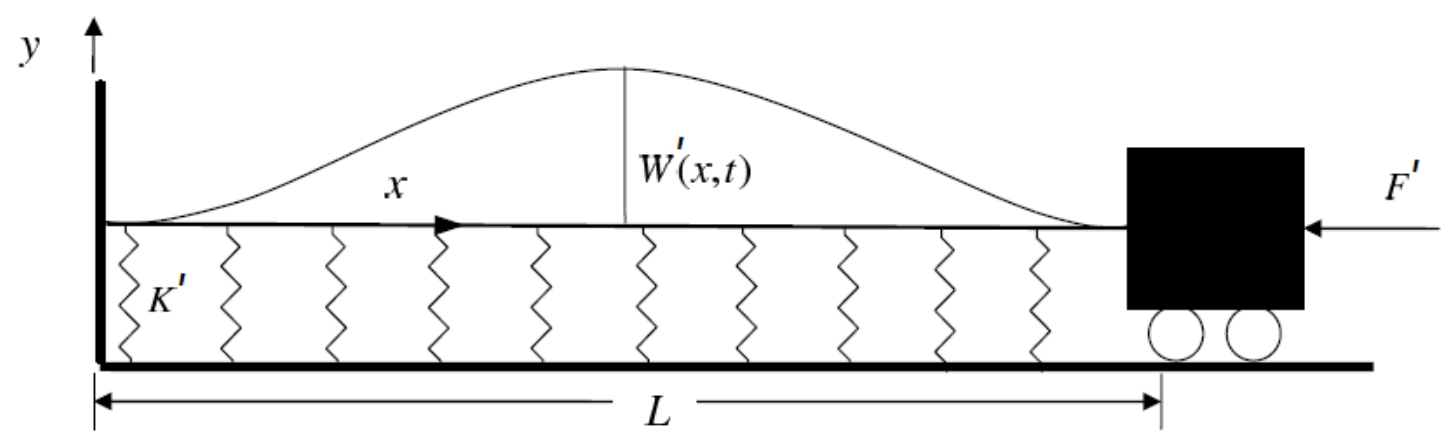

Figure 1 Schematic of an Euler-Bernoulli beam subjected to an axial load. 
The equation of motion including the effects of mid-plane stretching is given by:

$$
E I \frac{\partial^{4} W^{\prime}}{\partial X^{\prime 4}}+\mu \frac{\partial^{2} W^{\prime}}{\partial t^{\prime 2}}+\bar{F} \frac{\partial^{2} W^{\prime}}{\partial X^{\prime 2}}+C \frac{\partial W^{\prime}}{\partial t^{\prime}}+K^{\prime} W^{\prime}-\frac{E A}{2 L} \frac{\partial^{2} W^{\prime}}{\partial X^{\prime 2}} \int_{0}^{L}\left(\frac{\partial W^{\prime}}{\partial X^{\prime}}\right)^{2} d X^{\prime}=U\left(X^{\prime}, t^{\prime}\right)
$$

Where $C$ is the viscous damping coefficient, $K^{\prime}$ is a foundation modulus and $\mathrm{U}$ is a distributed load in the transverse direction.

Assume the non-conservative forces were equal to zero. Therefore Eq. (1) can be written as follows:

$$
E I \frac{\partial^{4} W^{\prime}}{\partial X^{\prime 4}}+\mu \frac{\partial^{2} W^{\prime}}{\partial t^{\prime 2}}+\bar{F} \frac{\partial^{2} W^{\prime}}{\partial X^{\prime 2}}+C \frac{\partial W^{\prime}}{\partial t^{\prime}}+K^{\prime} W^{\prime}-\frac{E A}{2 L} \frac{\partial^{2} W^{\prime}}{\partial X^{\prime 2}} \int_{0}^{L}\left(\frac{\partial W^{\prime}}{\partial X^{\prime}}\right)^{2} d X^{\prime}=0
$$

For convenience, the following nondimensional variables are used:

$$
X=\frac{X^{\prime}}{L}, \quad W=\frac{W^{\prime}}{R}, \quad t=t^{\prime} \sqrt{\frac{E I}{\mu L^{4}}}, \quad F=\frac{F^{\prime} L^{2}}{E I}, \quad K=\frac{K^{\prime} L^{4}}{E I}
$$

where $R=(I / A)^{0.5}$ is the radius of gyration of the cross section. As a result Eq. (2) can be written as follows:

$$
\frac{\partial^{4} W}{\partial X^{4}}+\frac{\partial^{2} W}{\partial t^{2}}+F \frac{\partial^{2} W}{\partial X^{2}}+K W-\frac{1}{2} \frac{\partial^{2} W}{\partial X^{2}} \int_{0}^{1}\left(\frac{\partial W}{\partial X}\right)^{2} d X=0
$$

Assuming $W(X, t)=\psi(X) \eta(t)$ where $\psi(X)$ is the first eigenmode of the beam (Tse, 1978) and applying the Galerkin method, the equation of motion is obtained as follows:

$$
\frac{d^{2} \eta(t)}{d t^{2}}+\alpha \eta(t)+\beta \eta(t)^{3}=0
$$

Where $a=a_{1}+a_{2} F+K$ and $a_{1}, a_{2}$ and $\beta$ are as follows:

$$
\alpha_{1}=\frac{\int_{0}^{1} \psi^{i v} \psi d x}{\int_{0}^{1} \psi^{2} d x}, \quad \alpha_{2}=\frac{\int_{0}^{1} \psi^{\prime \prime} \psi d x}{\int_{0}^{1} \psi^{2} d x}, \quad \beta=\frac{0.5 \int_{0}^{1}\left(\psi^{\prime \prime} \int_{0}^{1} \psi^{\prime 2} d x\right) \psi d x}{\int_{0}^{1} \psi^{2} d x}
$$

The Eq. (5) is the governing non-linear vibration of Euler-Bernoulli beams. The center of the beam subjected to the following initial conditions:

$$
\eta(0)=A, \quad \dot{\eta}(0)=0 .
$$


where $A$ denotes the non-dimensional maximum amplitude of oscillation.

\section{DESCRIPTION OF THE LAPLACE ITERATION METHOD}

Using the Laplace Transformation method an analytical approximated technique has been proposed to present an accurate solution for nonlinear differential equations. To clarify the basic ideas of proposed method consider the following second order differential equation,

$$
\ddot{u}(t)+N\{u(t)\}=0
$$

with artificial zero initial conditions and $N$ is the nonlinear operator. Adding and subtracting the term $\omega^{2} u(t)$, the Eq. (8) can be written in the form

$$
\ddot{u}(t)+\omega^{2} u(t)=L\{u(t)\}=f(u(t))
$$

where $L$ is the linear operator and

$$
f(u(t))=\omega^{2} u(t)-N\{u(t)\}
$$

Taking Laplace transform of both sides of the Eq. (9) in the usual way and using the homogenous initial conditions gives

$$
\left(s^{2}+\omega^{2}\right) U(s)=\mathfrak{I}\{f(u(t))\}
$$

where $S$ and $\mathfrak{I}$ are the Laplace variable and operator, correspondingly. Therefore it is obvious that

$$
U(s)=\mathfrak{I}\{f(u(t))\} G(s)
$$

where

$$
G(s)=\frac{1}{s^{2}+\omega^{2}}
$$


Now, implementing the Laplace inverse transform of Eq. (12) and using the Convolution theorem offer

$$
u(t)=\int_{0}^{t} f(u(\tau)) g(t-\tau) d \tau
$$

where

$$
g(t)=\mathfrak{I}^{-1}\{G(s)\}=\frac{1}{\omega} \sin (\omega t)
$$

Substituting Eq. (10) and (15) into (14) gives

$$
u(t)=\int_{0}^{t}\left(\omega^{2} u(\tau)-N\{u(\tau)\}\right) \frac{1}{\omega} \sin (\omega(t-\tau)) d \tau
$$

Now, the actual initial conditions must be imposed. Finally the following iteration formulation can be used (Hesameddini and Latifizadeh, 2009)

$$
u_{n+1}=u_{0}+\frac{1}{\omega} \int_{0}^{t}\left(\omega^{2} u_{n}(\tau)-N\left\{u_{n}(\tau)\right\}\right) \sin (\omega(t-\tau)) d \tau
$$

Knowing the initial approximation $u_{0}$, the next approximations $u_{n}, n>0$ can be determined from previous iterations. Consequently, the exact solution may be obtained by using:

$$
u=\lim _{n \rightarrow \infty} u_{n}
$$

In this method, the problems are initially approximated with possible unknowns and it can be applied in non-linear problems without linearization or small parameters. The approximate solutions obtained by the proposed method rapidly converge to the exact solution. 


\section{IMPLEMENTATION OF LAPLACE ITERATION METHOD}

Rewriting Eq. (5) in the standard form of Eq. (9) results in the following equation:

$$
\ddot{\eta}(t)+\omega^{2} \eta(t)=f(\eta(t)), \quad \eta(0)=A, \quad \dot{\eta}(0)=0 .
$$

Where

$$
\begin{aligned}
& f(\eta(t))=\omega^{2} \eta(t)-N\{\eta(t)\}, \\
& N\{\eta(t)\}=\alpha \eta(t)+\beta \eta(t)^{3}
\end{aligned}
$$

Applying the proposed method, the following iterative formula is formed as:

$$
\eta_{n+1}(t)=\eta_{0}(t)+\frac{1}{\omega} \int_{0}^{t} f\left(\eta_{n}(\tau)\right) \sin (\omega(t-\tau)) d \tau
$$

Eq. (19) will be homogeneous, when $f(\eta(t))$ has a value of zero. So, its homogeneous solution

$$
\eta_{0}(t)=A \cos (\omega t)
$$

is considered as the zero approximation for using in iterative Eq.(21).

To obtain frequency of the system ( $\omega)$, from Eq. (5), we have:

$$
\begin{gathered}
\ddot{\eta}=-\alpha \eta-\beta \eta^{3} \\
\ddot{\eta}=-\alpha A \cos (\omega t)-\beta A^{3} \cos ^{3}(\omega t)
\end{gathered}
$$

Integrating twice yields:

$$
\eta_{1}=\frac{-7 A^{3} \beta-A^{3} \beta \cos ^{3}(\omega t)+6 A^{3} \beta \cos (\omega t)-9 A \alpha+9 A \alpha \cos (\omega t)}{9 \omega^{2}}
$$

Equating the coefficients of $\cos (\omega t)$ in $\eta_{0}$ and $\eta_{1}$, the first approximation of the frequency can be expressed as: 


$$
\omega_{L I M}=\sqrt{\alpha+\frac{3}{4} \beta A^{2}}
$$

Substituting Eq. (22) into (21) and eliminating the secular terms which is the coefficient of $\cos (\omega t)$ in forcing function $f(\eta)$ results in:

$\eta_{1}(t)=A \cos (\omega t)-\frac{A^{3} \beta(\cos (\omega t)-\cos (3 \omega t))+12 A^{3} \beta \omega t \sin (\omega t)-16 A \omega^{3} t \sin (\omega t)+16 A \alpha \omega t \sin (\omega t)}{32 \omega^{2}}$

where $\omega$ is evaluated from Eq. (26). In the same manner, the rest of the components of the iteration formula can be obtained.

\section{DESCRIPTION OF THE HE'S VARIATIONAL APPROACH}

In recent years, a heuristic variational approach, based on the method of energy balance has been introduced to solve nonlinear systems requiring no small parameters. This method, known as He's variational method, can be easily implemented and yields to highly accurate results. In this method, a variational principle for the nonlinear oscillations is established, then a Hamiltonian is constructed, from which the angular frequency can be obtained by a lacation method. To explain the method precisely, consider the following generalized nonlinear oscillations without forced terms:

$$
u^{\prime \prime}+\omega^{2} u+\varepsilon f(u)=0, \quad u(0)=A, \quad u^{\prime}(0)=0
$$

where $f$ is a nonlinear function of $u, u^{\prime}$ and $u^{\prime \prime}$.

For simplicity, if function $f$ depends on $u$ only, its variational functional can be obtained as:

$$
J(u)=\int_{0}^{t}\left\{-\frac{u^{\prime}}{2}+\omega^{2} \frac{u^{2}}{2}+\varepsilon F(u)\right\} d t
$$

where $F$ is the potential, $d F / d u=f$.

Its Hamiltonian reads

$$
H=-\frac{u^{\prime}}{2}+\omega^{2} \frac{u^{2}}{2}+\varepsilon F(u)
$$

which should be an invariant during the oscillation. With the invariant, we can apply the location method to identify the frequency by suitable choice of trial functions (He, 2006). If, by chance, the 
exact solution had been chosen as the trial function, then it would be possible to make the residual zero for all values of $t$ by appropriate choice of $\omega$. From this point of view, He's variational method is completely different from the classical variational methods, but has little likeness to Ritz method. Because, Ritz method is only valid for fixed domain, but in He's variational method, the integral boundary is not fixed. So He's functional formulation can be called as variational functional with movable domain. Therefore, He's variational approach is quite different from the classic Ritz method in calculus of variation. Following the approach adopted by the Öziş and Yildirim, 2006) in a previous publication He's method has been used to determine the periodic solutions and the periods of strongly nonlinear systems given below in subsequent three examples (Öziş and Yildirim, 2006). More recently, He's method also adopted by D'Acunto for self-excited systems (D'Acunto, 2006a; $2006 \mathrm{~b})$.

\section{IMPLEMENTATION OF HE'S VARIATIONAL APPROACH}

The governing equation of the system is:

$$
\frac{d^{2} \eta(t)}{d t^{2}}+\alpha \eta(t)+\beta \eta(t)^{3}=0, \quad \eta(0)=A, \quad \dot{\eta}(0)=0
$$

We can, now, easily obtain the following variational formulation:

$$
J(\eta)=\int_{0}^{T}\left\{-\frac{\eta^{\prime}}{2}+\alpha \frac{\eta^{2}}{2}+\beta \frac{\eta^{4}}{4}\right\} d t
$$

By He's method; we search the solution in the form:

$$
\eta(t)=A \cos (\omega t)
$$

Substituting Eq.(33) into Eq.(32) results in

$$
J(\eta)=\int_{0}^{\frac{2 \pi}{\omega}}\left\{-\frac{1}{2} A^{2} \omega^{2} \sin ^{2}(\omega t)+\frac{1}{2} \alpha A^{2} \cos ^{2}(\omega t)+\frac{1}{4} \beta A^{4} \cos ^{4}(\omega t)\right\} d t
$$

or

$$
J(\eta)=0.1963495409 \frac{A^{2}\left(-8 \omega^{2}+3 \beta A^{2}\right)}{\omega} .
$$


Making $J$ stationary with respect to $A$, according to He's method (He, 2007), we obtain:

$$
\frac{\partial J}{\partial A}=0.1963495409 \frac{2 A\left(-8 \omega^{2}+8 \alpha+3 \beta A^{2}\right)+A^{2}(6 \beta A)}{\omega}=0
$$

or simplifying

$$
-16 A \omega^{2}+16 A \alpha+12 \beta A^{3}=0
$$

So the frequency can be approximated as:

$$
\omega_{V A}=\sqrt{\alpha+\frac{3}{4} \beta A^{2}}
$$

and zero-order approximate solution from Eq.(33) is:

$$
\eta(t)=A \cos \left(\sqrt{\alpha+0.75 \beta A^{2}} t\right)
$$

\section{RESULTS AND DISSCUSIONS}

In this stage, nonlinear responses of a clamped-clamped buckled beam are proposed. Mathematically, the beam is modeled by a partial differential equation possessing cubic non-linearity because of mid plane stretching. Governing non-linear partial differential equation of Euler- Bernoulli's beam is reduced to a single non-linear ordinary differential equation using Galerkin method. The frequency of both methods is exactly the same and transverse vibration of the beam center is illustrated versus amplitude and time. The effects of non-dimensional maximum amplitude of oscillation, A, on the time marching solutions for the motion equation is investigated in Table 1.

In order to verify the precision of the methods, current results were compared with Runge-Kutta 4th order method in Table 1. It is observable that our results are in excellent agreement with the results provided by Runge-Kutta 4 th order. The behavior of $\eta(A, t)$ obtained by LIM and VA at $a=\pi$ and $\beta=0.15$ is shown in Figs. 2 and 3. The comparison of the dimensionless deflection versus time for results obtained from LIM, VA and Runge-Kutta 4 th order has been depicted in Fig. 4 for $a=\pi$ and $\beta=0.15$, with maximum deflection at the center of the beam equal to five $(A=5)$. 
Table 1 Comparison between LIM \& VA with time marching solution for the motion equation (5), when $t=0.5(s)$, $\alpha=\beta=1$

\begin{tabular}{cccccc}
\hline $\mathrm{A}$ & LIM & VA & RKF & ERROR(LIM) & ERROR(VA) \\
\hline \hline 0.01 & 0.008775710 & 0.008775735 & 0.008775693 & -0.000000017 & -0.000000042 \\
0.1 & 0.087643206 & 0.087668378 & 0.087643042 & -0.000000168 & -0.000025336 \\
0.2 & 0.174597455 & 0.174797831 & 0.174597438 & -0.000000017 & -0.000200393 \\
0.3 & 0.260180487 & 0.260851146 & 0.260182593 & -0.000002106 & -0.000668553 \\
0.4 & 0.343723299 & 0.345294531 & 0.343733783 & 0.000010484 & -0.001560748 \\
0.5 & 0.424576467 & 0.427599316 & 0.424609486 & 0.000033019 & -0.002989830 \\
1 & 0.767843030 & 0.789109858 & 0.768790533 & -0.000947503 & -0.020319325 \\
10 & -2.960606970 & -3.461741578 & -3.700321826 & -0.739714856 & -0.238580248 \\
\hline \hline
\end{tabular}

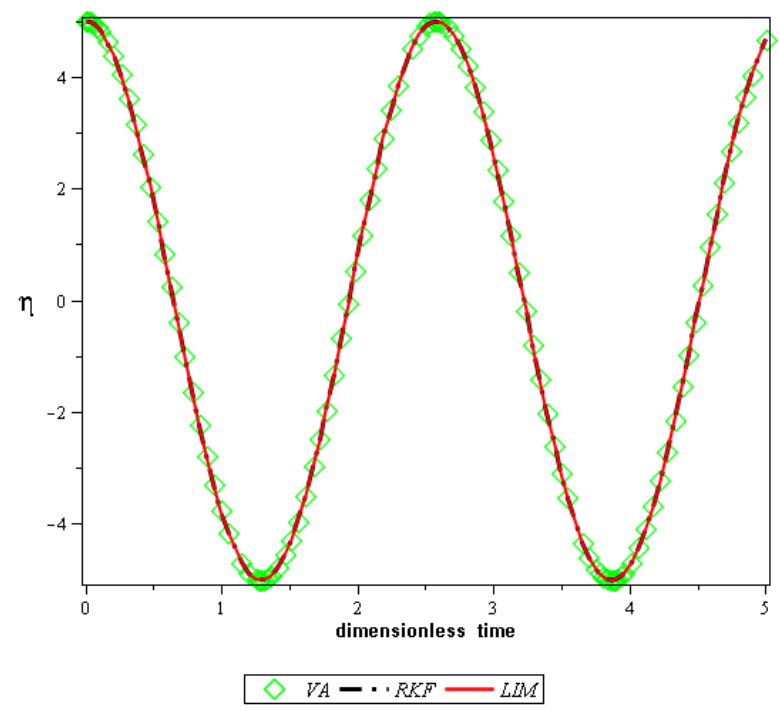

Figure 2 Comparison between LIM and VA deflection versus time $\alpha=\pi$ and $\beta=0.15$ and $A=5$ with Runge-Kutt 4th order.

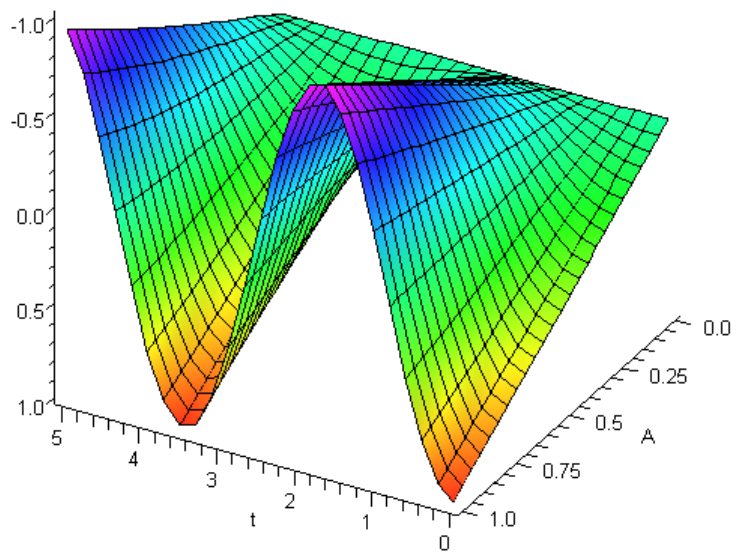

Figure 3 LIM deflection at $\alpha=\pi$ and $\beta=0.15$

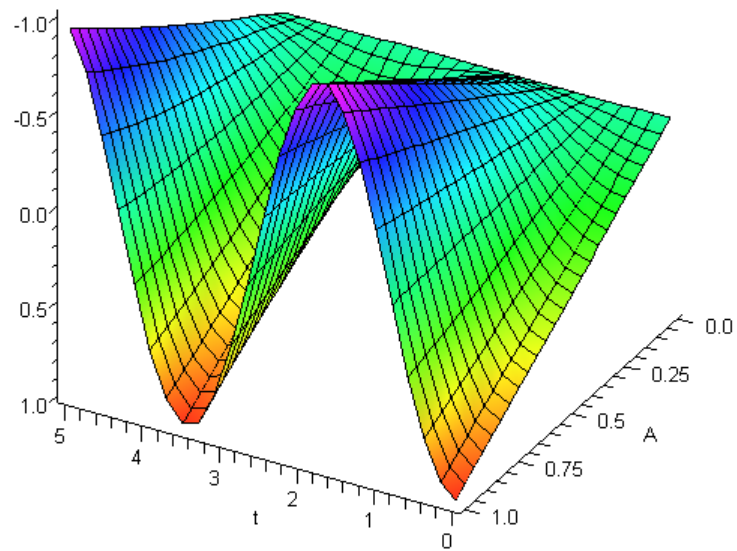

Figure 4 VA deflection at $\alpha=\pi$ and $\beta=0.15$ 


\section{CONCLUSIONS}

In this study, nonlinear responses of a clamped-clamped buckled beam are established to verify the vibrational behaviors of beam. Variational approach and efficient approximate method (LIM) is employed to derive the nonlinear responses of a clamped-clamped buckled beam. The frequency of both methods is exactly the same and transverse vibration of the beam center is illustrated versus amplitude and time. Comparing with numerical Results, it is shown that the approximate analytical solutions are in very good agreement with the corresponding solutions.

\section{References}

Barari, A., Kaliji, H. D., Ghadimi, M., Domairry, G., (2011). Non-linear vibration of Euler-Bernoulli beams, Latin American Journal of Solids and Structures 8(2):139-148.

Bayat, Mahmoud., Pakar, Iman., Bayat, Mahdi., (2011). Analytical study on the vibration frequencies of tapered beams, Latin American Journal of Solids and Structures 8(2):149-162.

Boukhalfa, Abdelkrim., Hadjoui, Abdelhamid., (2010). Free vibration analysis of an embarked rotating composite shaft using the hp-version of the FEM, Latin American Journal of Solids and Structures 7:105-141.

Burgreen, D., (1984). Free vibration of a pin-ended column with constant distance between pin ends, Journal of Applied Mechanics 18:135-139.

Da Silva, J. C. R. Á., Beck, A. T., da Rosa, E., (2009). Solution of the stochastic beam bending problem by Galerkin method and the Askey-Wiener scheme, Latin American Journal of Solids and Structures 6(1):51-72.

D'Acunto. M. (2006a). Self-exited systems: Analytical determination of limit cycles, Chaos, Solitons and Fractals, 30:719-724, 2006.

D'Acunto. M. (2006b). Determination of limit cycles for a modified van der Pol oscillator, Mechanics Research Communications 33:93-98.

Fereidoon, A., Rostamiyan, Y., Akbarzade, M., Ganji, D. D., (2010). Application of He's homotopy perturbation method to nonlinear shock damper dynamics, Archive of Applied Mechanics 80(6):641-649.

Ganji, D. D. (2012). A semi-Analytical technique for non-linear settling particle equation of Motion, Journal of Hydro-environment Research 6(4): 323-327.

Ganji, S. S., Barari, A., Ganji, D. D., (2011). Approximate analysis of two-mass-spring systems and buckling of a column, Computers \& Mathematics with Applications 61(4):1088-1095.

Ghasempour, S., Vahidi, J., Nikkar A., Mighani, M., (2013). Analytical Approach to Some Highly Nonlinear Equations by Means of the RVIM, Research Journal of Applied Sciences, Engineering and Technology, 5(1): 339-345.

Ghotbi, Abdoul.R., Bararnia, H., Domairry, G., Barari, A., (2009). Investigation of a powerful analytical method into natural convection boundary layer flow, Communications in Nonlinear Science and Numerical Simulation 14 (5): 2222-2228.

He, J. H. (2006). Some asymptotic methods for strongly nonlinear equations, International Journal of modern Physics B 20(10):1141-1199.

He, J. H. (2007). Variational approach for nonlinear oscillators, Chaos, Solitons and Fractals 34:1430-1439.

Hesameddini, E., Latifizadeh, H., (2009). Reconstruction of Variational Iteration Algorithms using the Laplace Transform, Int J Nonlinear Sci Numer Simul 10(10):1365-1370. 
Hsu, J.C., Lai, H.Y., Chen, C.K., (2008). Free Vibration of non-uniform Euler-Bernoulli beams with general elastically end constraints using Adomian modified decomposition method, Journal of Sound and Vibration 318: 965-981.

Khan, Y., Taghipour, R., Fallahian, M., Nikkar, A., (2012). A new approach to modified regularized long wave equation, Neural Computing and Applications doi:10.1007/s00521-012-1077-0

Lai, H.Y., Hsu, J.C., Chen, C.K., (2008). An innovative eigenvalue problem solver for free vibration of EulerBernoulli beam by using the Adomian Decomposition Method, Computers and Mathematics with Applications, 56: 3204-3220.

Liu, Y., Gurram, C. S., (2009). The use of He's variational iterationmethod for obtaining the free vibration of an Euler-Beam beam, Matematical and Computer Modelling 50:1545-1552.

Rafieipour, H., Lotfavar, A., Mansoori, M.H., (2012). New Analytical Approach to Nonlinear Behavior Study of Asymmetrically LCBs on Nonlinear Elastic Foundation under Steady Axial and Thermal Loading. Latin American Journal of Solids and Structures 9(5):531-545.

Rao, S. S. (2007). Vibraton of Continuous Systems. John Wiley \& Sons, Inc., Hoboken, New Jersey.

Tse, F. S., Morse, I. E., Hinkle, R. T., (1978) Mechanical Vibrations. Theory and Applications. Allyn and Bacon Inc, second edition.

Nikkar, A., Mighani, Z., Saghebian, S.M., Nojabaei, S.B., Daie, M., (2013). Development and Validation of an Analytical Method to the Solution of Modelling the Pollution of a System of Lakes, Research Journal of Applied Sciences, Engineering and Technology 5(1):296-302.

Öziş, T., Yildirim A., (2006). Determination of frequency-amplitude relation for Duffing-Harmonic oscillator by the energy balance method, Special Issue of Computers and Mathematics with Applications 54(7):1184-1187.

Pirbodaghi, T., Ahmadian, M.T., Fesanghary, M., (2009). On the homotopy analysis method for non-linear vibration of beams, Mechanics Research Communications 36:143-148. 